\title{
Optimizing the fast Rydberg quantum gate
}

\author{
M. S. Safronova, Carl J. Williams, and Charles W. Clark \\ Physics Laboratory, National Institute of Standards and Technology, Technology Administration, \\ U.S. Department of Commerce, Gaithersburg, Maryland 20899-8410
}

(Dated: December 2, 2018)

\begin{abstract}
The fast phase gate scheme, in which the qubits are atoms confined in sites of an optical lattice, and gate operations are mediated by excitation of Rydberg states, was proposed by Jaksch et al. Phys. Rev. Lett. 85, 2208 (2000). A potential source of decoherence in this system derives from motional heating, which occurs if the ground and Rydberg states of the atom move in different optical lattice potentials. We propose to minimize this effect by choosing the lattice photon frequency $\omega$ so that the ground and Rydberg states have the same frequency-dependent polarizability $\alpha(\omega)$. The results are presented for the case of $\mathrm{Rb}$.
\end{abstract}

PACS numbers: 03.67.Lx, 32.10.Dk, 32.80.Rm

Recently, a number of schemes for quantum computation with neutral atoms have been proposed [1, 2, 3, 4, 5, 6, 7, 8, 9. 9. In those schemes, qubits are realized as internal states of neutral atoms trapped in optical lattices or magnetic microtraps. The two-qubit quantum gates are realized using controlled cold collisions [1, 2, 3, , 4, controlled dipole-dipole interactions 肘, 6, 7], or by conditional excitations of atoms into the Rydberg states by a series of laser pulses [8, 9]. This approach to quantum computation has many advantages, such as scalability, possible massive parallelism, long decoherence times of the internal states of the atoms, flexibility in controlling atomic interactions, and well-developed experimental techniques. As extensive experimental studies of the feasibility of using neutral atoms for quantum computation are under way, more detailed theoretical investigation of the various schemes is needed. In this work, we investigate optimization of the quantum gate scheme realized by excitations to Rydberg states [8]. We refer to this scheme below as the Rydberg gate. The choice of this particular scheme results from its potential for fast (sub-microsecond) gate operations.

In the Rydberg gate scheme, the basic qubit is based on two ground hyperfine states of neutral atoms confined in an optical lattice. A two-qubit phase gate may be realized by conditionally exciting two atoms to low-lying Rydberg states. Different versions of the scheme have been proposed [8]. In those schemes, either one or both atoms may occupy the Rydberg state for much of the duration of the gate operation. However, an atom in a Rydberg state will, in general, move in different optical lattice potential than that experienced by the ground state. Therefore, the vibrational state of the atom in the lattice may change after the gate operation is completed, leading to decoherence due to motional heating. The optical potential for a given state depends on its ac polarizability, so we can seek to minimize this motional heating effect by the choice of a particular Rydberg state or of the lattice photon frequency, $\omega$. In this paper, we describe a method for accomplishing this by matching the frequency-dependent polarizabilities, $\alpha(\omega)$, of the atomic ground state and Rydberg state. The results are pre- sented for case of Rb; however, the approach used here is applicable for logic gates with other alkali-metal atoms.

In this work, we calculate polarizabilities of the $\mathrm{Rb}$ atom in its ground state, $\alpha_{5 s}(\omega)$, and in various Rydberg $n s$ states $\alpha_{n s}(\omega)$. We demonstrate that there exists a lattice frequency $\omega$ such that

$$
\alpha_{5 s}(\omega) \approx \alpha_{n s}(\omega)
$$

This finding relies upon the fact 10 that the frequencydependent polarizabilities of alkali Rydberg states are close to that of a free electron, $\alpha_{\text {free }}(\omega)=-e^{2} / m_{\mathrm{e}} \omega^{2}$, where $e$ is the elementary charge in statcoulombs, and $m_{\mathrm{e}}$ is the electron mass. For the optical frequencies treated in this paper, $\alpha_{n l}(\omega)$ for Rydberg states is comparable in magnitude to $\alpha_{5 s}(0)$, but it has a negative sign. As a function of $\omega, \alpha_{5 s}(\omega)$ increases as $\omega$ increases from zero, but changes sign when $\omega$ exceeds the first resonance frequency. We show that it will always be possible to find a frequency for which the Rydberg and ground-state polarizabilities are equal. An optical lattice constructed with light of that frequency will have well depths characteristic of far-off-resonance traps.

An alternative approach to polarizability matching of ground and Rydberg states is to find Rydberg states with resonant frequencies close to those of the ground state. In such cases the polarizabilities can be matched at nearresonant frequencies, resulting in a tighter trap for a given laser intensity. Such possibilities can be identified by a straightforward search of tabulated atomic energy levels, and they turn out to be rare. The two cases of interest in $\mathrm{Rb}$ are discussed at the end of this paper.

We begin with a description of our calculation of the polarizabilities. It is convenient for this purpose to use the system of atomic units, a.u., in which $e, m_{\mathrm{e}}, 4 \pi \epsilon_{0}$ and the reduced Planck constant $\hbar$ have the numerical value 1. Polarizability in a.u. has the dimensions of volume, and its numerical values presented here are thus measured in units of $a_{0}^{3}$, where $a_{0} \approx 0.052918 \mathrm{~nm}$ is Bohr radius. The atomic units for $\alpha$ can be be converted to SI units via $\alpha / h\left[\mathrm{~Hz} /(\mathrm{V} / \mathrm{m})^{2}\right]=2.48832 \times 10^{-8} \alpha$ [a.u.], where the conversion coefficient is $4 \pi \epsilon_{0} a_{0}^{3} / h$ and Planck constant $h$ is factored out. The atomic unit of frequency $\omega$ 
is $E_{h} / \hbar \approx 4.1341 \times 10^{16} \mathrm{~Hz}$, where $E_{h}$ is Hartree energy.

The valence contribution to the dynamic polarizability for an alkali atom in an $n s$ state can be calculated using the formula (in a.u.) 14]

$$
\begin{aligned}
\alpha_{n s}(\omega) & =\frac{1}{3} \sum_{n^{\prime}}\left(\frac{\left(E_{n^{\prime} p_{1 / 2}}-E_{n s}\right)\left\langle n^{\prime} p_{1 / 2}\|D\| n s\right\rangle^{2}}{\left(E_{n^{\prime} p_{1 / 2}}-E_{n s}\right)^{2}-\omega^{2}}\right. \\
& \left.+\frac{\left(E_{n^{\prime} p_{3 / 2}}-E_{n s}\right)\left\langle n^{\prime} p_{3 / 2}\|D\| n s\right\rangle^{2}}{\left(E_{n^{\prime} p_{3 / 2}}-E_{n s}\right)^{2}-\omega^{2}}\right)
\end{aligned}
$$

where $D$ is the electric dipole operator and $E_{i}$ is the energy of the state $i$. In this formula, $\omega$ is assumed to be at least several linewidths off resonance with the corresponding transition. This condition is satisfied for the frequencies considered in this work, as the lattice has to be significantly detuned from the resonance to minimize spontaneous emission. The core contribution to the polarizability, calculated in the Hartree-Fock (HF) approximation, is found to be small $\left(9.3 a_{0}^{3}\right)$ and is weakly dependent on $\omega$ in the frequency range considered here. The static value for the polarizability of $\mathrm{Rb}^{+}$calculated in the random phase approximation (RPA) [11, 12 is $9.1 a_{0}^{3}$, close to the value of $9.0 a_{0}^{3}$ obtained from by Johansson [13 from analysis of the observed term values of nonpenetrating Rydberg states. The accuracy of the core contribution is estimated to be $5 \%$ in Ref. 14. We use the RPA value for the core polarizability of $\mathrm{Rb}^{+}$as a baseline, and adjust it to account for valence electron and the frequency dependence by using HF calculations. The RPA and HF values differ by only $2 \%$.

In calculating the valence contribution, the term with $n=5$ dominates the the sum over states, and the contributions from higher values of $n$ converge rapidly for all values of $\omega$ considered in this work. The $5 s-5 p$ matrix elements were measured in Ref. [15] and the $5 s-n p$ matrix elements with $n=6,7,8$ were calculated using single-double all-order method in Refs. [14, 16. We have conducted the calculation of the ground $(5 s)$ state $\alpha_{5 s}(\omega)$ in $\mathrm{Rb}$, including terms with $n=5,6,7$, and 8 using matrix elements from Refs. [15, 16] and experimental energies from 17]. The contributions to the ground state polarizability from states with $n^{\prime}=8-\infty$, including the continuum, and from terms with $n=2,3,4$ are estimated to be very small $\left(\begin{array}{llll}0.2 & a_{0}^{3}\end{array}\right.$ and $-0.3 a_{0}^{3}$, respectively). The results for the values of $\omega$ near first two resonances, $5 s-5 p_{1 / 2}$ and $5 s-5 p_{3 / 2}$ are listed in Table II. As expected, the value of the frequency-dependent polarizability varies very rapidly in the vicinity of the $5 s-5 p_{1 / 2}$ and $5 s-5 p_{3 / 2}$ resonances. The second set of values of $\alpha_{5 s}(\omega)$ listed in column four illustrates the existence of the $\alpha_{5 s}(\omega)=0$ point between the $5 s-5 p_{1 / 2}$ and $5 s-5 p_{3 / 2}$ resonances. The next resonance occurs at $\omega=E_{5 s}-E_{6 p_{1 / 2}}$.

Molof et al. [18 measured $\alpha_{5 s}(0)$ to be $47.3 \pm 0.9 \AA^{3}$, equivalent to $319 \pm 6 a_{0}^{3}$. Our calculated value agrees with this result to within the experimental uncertainty. The static polarizability of the ground state of $\mathrm{Rb}, \alpha_{5 s}(0)$,
TABLE I: Dynamic polarizabilities $\alpha(\omega)$ of the ground state of $\mathrm{Rb}$ in units of $a_{0}^{3} ; \omega$ is in a.u. $\left(E_{h} / \hbar\right)$.

\begin{tabular}{lrrrrr}
\hline \hline$\omega$ & \multicolumn{1}{c}{$\alpha$} & \multicolumn{1}{c}{$\omega$} & $\alpha$ & \multicolumn{1}{c}{$\omega$} & \multicolumn{1}{c}{$\alpha$} \\
\hline 0 & $318.5(6)$ & 0.0576500 & $-815(23)$ & 0.0578 & $3931(20)$ \\
0.02000 & $360.0(7)$ & 0.0576645 & $-290(23)$ & 0.058 & $10755(31)$ \\
0.04000 & $597.8(8)$ & 0.0576700 & $-97(22)$ & 0.059 & $-11548(16)$ \\
0.04298 & $693.5(9)$ & 0.0576722 & $-21(22)$ & 0.060 & $-4737(5)$ \\
0.05000 & $1211(1)$ & 0.0576728 & $0(22)$ & 0.065 & $-1206(1)$ \\
0.05500 & $3132(3)$ & 0.0576734 & $21(22)$ & 0.070 & $-667.1(9)$ \\
0.05700 & $13854(23)$ & 0.0576800 & $246(22)$ & 0.080 & $-330.3(8)$ \\
0.05750 & $-9311(57)$ & 0.0576900 & $581(22)$ & 0.090 & $-205.9(8)$ \\
0.05760 & $-2869(29)$ & 0.0577000 & $907(21)$ & 0.100 & $-138(1)$ \\
\hline \hline
\end{tabular}

was previously calculated using the techniques described above in Ref. [11]. The general behavior of the frequencydependent polarizability of the ground state of $\mathrm{Rb}$ has been investigated previously in [19. A result for one particular value of $\omega=0.04298$ a.u., corresponding to $\lambda=1.06 \mu \mathrm{m}$ 20], has been calculated in the same work, using a model potential method. We compare our value of the ground state dynamic polarizability for this frequency with the result of Marinescu et al. [19. Our result $\alpha=693.5(9) a_{0}^{3}$, listed in Table If, is in good agreement with the result $\alpha=711.4 a_{0}^{3}$ from Ref. [19]. It is just outside the range of uncertainty of the value $\alpha=769 \pm 61 a_{0}^{3}$ inferred by Bonin and Kadar-Kallen from an atomic deflection experiment 21]. A subsequent publication 22] will provide further details of the polarizability calculations, as well as calculation of the radiative widths of $n p$ levels to quantify the behavior of the ground state polarizability near the resonances.

Second, we calculate the dynamic polarizabilities of the Rydberg states for a specific value of the lattice photon frequency $\omega$ and then investigate the dependence of polarizability on $\omega$ for several Rydberg states. The most likely realization of the Rydberg gate scheme involves two-photon transitions from the ground state to either $n s$ or nd states. For clarity, we calculate the polarizabilities of the $n s$ states in this work. To make an estimate of the $n s$ Rydberg state polarizability $\alpha_{n s}(\omega)$ we first calculate relevant matrix elements and energies in Hartree-Fock approximation. The HF calculations are done on a non-linear grid, and the question of the numerical stability of the calculation of the properties of the Rydberg states was investigated by conducting the calculation with several different grids to insure the stability of the results. We use the resulting matrix elements to calculate frequency-dependent polarizabilities of the $n s$ states in $\mathrm{HF}$ approximation. The results for the $n s$ state polarizabilities with $\omega=0.0576645$ a.u. are listed in Table III in the columns labeled $\alpha_{\mathrm{HF}}$. This frequency corresponds to the value of the $\alpha_{5 s}(\omega)=-290 a_{0}^{3}$ and to a detuning approximately $1 / 3$ of the distance between the two resonances; $\alpha_{\text {free }}(\omega)=-301 a_{0}^{3}$ at this frequency.

We observe that there is no significant change of the 
TABLE II: Dynamic polarizabilities $\alpha_{n s}(\omega)$ (in units of $a_{0}^{3}$ ) for $\mathrm{Rb}, \omega=0.0576645$ a.u., i.e. $\lambda=790 \mathrm{~nm}$.

\begin{tabular}{ccccc}
\hline \hline$N$ & $\alpha_{\mathrm{HF}}$ & $\alpha$ & $N$ & $\alpha_{\mathrm{HF}}$ \\
\hline 8 & -304 & $-295^{a}$ & 14 & -286 \\
9 & -292 & & 15 & -285 \\
10 & -289 & & 16 & -284 \\
11 & -288 & & 17 & -282 \\
12 & -287 & & 18 & -280 \\
13 & -287 & & 19 & -277 \\
\hline \hline
\end{tabular}

${ }^{a}$ High-accuracy value obtained using experimental energies and all-order matrix elements for the dominant terms with $n^{\prime}=7,8$.

polarizability values after $n \geq 8$. The summation over $n$ in Eq. (2) is truncated at $n=23$. The contribution of the states with $n>23$ and continuum is evaluated by carrying out a calculation of the polarizability of the $10 s$ state with B-spline basis set [23]. The summation over the entire basis set yields the result -292 a.u. which differs from the value in Table II by only $1 \%$. To evaluate the uncertainty of the HF approximation further we repeat the calculations using HF matrix elements and experimental energies from Ref. [17] in Eq. (22). We find a substantial, from $10 \%$ to $30 \%$, difference between these two approximations despite only a few percent differences between Hartree-Fock and experimental $\left[E_{n s}-E_{n p}\right]$ energies. Such a large discrepancy is explained by severe cancellations of the different terms in the sum of Eq. (2). As an illustration, we list the contributions to the polarizability of the $15 s$ state from several dominant terms together with the values for the corresponding dipole matrix elements $\langle n p\|D\| 15 s\rangle$, energy differences $\delta E=E_{n p}-E_{15 s}$, and denominators of Eq. (2) in Table III. We find that the dominant contributions come from $n=14$ and $n=15$ terms which have different sign owing to the sign change in the energy differences. We also note that the denominator $(\delta E)^{2}-\omega^{2}$ is completely dominated by the $\omega^{2}$ term, and, therefore, nearly identical for all of the contributions. We also note the cancellations of the smaller pairs of terms with $n=13,16$ and $n=12,17$.

The cancellations of dominant contributions have also been observed in the calculations of the vector transition polarizability $\beta$ and parity-nonconserving amplitude in Cs and Fr ( see, for example 14, 16] and references therein) when calculated using similar "directsummation" method. Generally, the values obtained using sets of data of the consistent accuracy are more reliable in such cases, so we use HF data below. The accuracy of the values can be increased by estimating correlation correction contributions to the values of the dipole matrix elements. We conduct such calculation for $\alpha_{8 s}(\omega)$ for the same frequency as data in Table II using experimental energies and high-accuracy all-order matrix elements for the dominant terms with $n^{\prime}=7,8$. The result $\left(-295 a_{0}^{3}\right)$ is listed in Table $\mathbb{1}$ and agrees very well with
TABLE III: Contributions to the dynamic polarizability of the $15 s$ state of Rb in a.u., $\omega=0.0576645$ a.u., $\delta E=E_{n p}-E_{15 s}$, $D=\langle n p\|D\| 15 s\rangle$. The contributions from different terms are given in column $\alpha$ (contr). The accumulated values are given in column $\alpha(\operatorname{acc})$.

\begin{tabular}{crrrrr}
\hline \hline$n p$ & \multicolumn{1}{c}{$D$} & \multicolumn{1}{c}{$\delta E$} & $(\delta E)^{2}-\omega^{2}$ & $\alpha($ contr $)$ & $\alpha($ acc $)$ \\
\hline $12 p_{1 / 2}$ & -5.9 & -0.00215 & -0.00332 & 8 & 28 \\
$12 p_{3 / 2}$ & -8.2 & -0.00214 & -0.00332 & 14 & 42 \\
$13 p_{1 / 2}$ & -15.6 & -0.00111 & -0.00332 & 27 & 69 \\
$13 p_{3 / 2}$ & -21.7 & -0.00110 & -0.00332 & 52 & 122 \\
$14 p_{1 / 2}$ & -111.8 & -0.00034 & -0.00333 & 424 & 545 \\
$14 p_{3 / 2}$ & -161.0 & -0.00033 & -0.00333 & 858 & 1403 \\
$15 p_{1 / 2}$ & 144.0 & 0.00026 & -0.00333 & -536 & 867 \\
$15 p_{3 / 2}$ & 201.2 & 0.00026 & -0.00333 & -1072 & -204 \\
$16 p_{1 / 2}$ & 16.1 & 0.00073 & -0.00332 & -19 & -223 \\
$16 p_{3 / 2}$ & 23.9 & 0.00073 & -0.00332 & -42 & -265 \\
$17 p_{1 / 2}$ & 6.4 & 0.00110 & -0.00332 & -5 & -270 \\
$17 p_{3 / 2}$ & 9.7 & 0.00110 & -0.00332 & -10 & -280 \\
\hline \hline
\end{tabular}

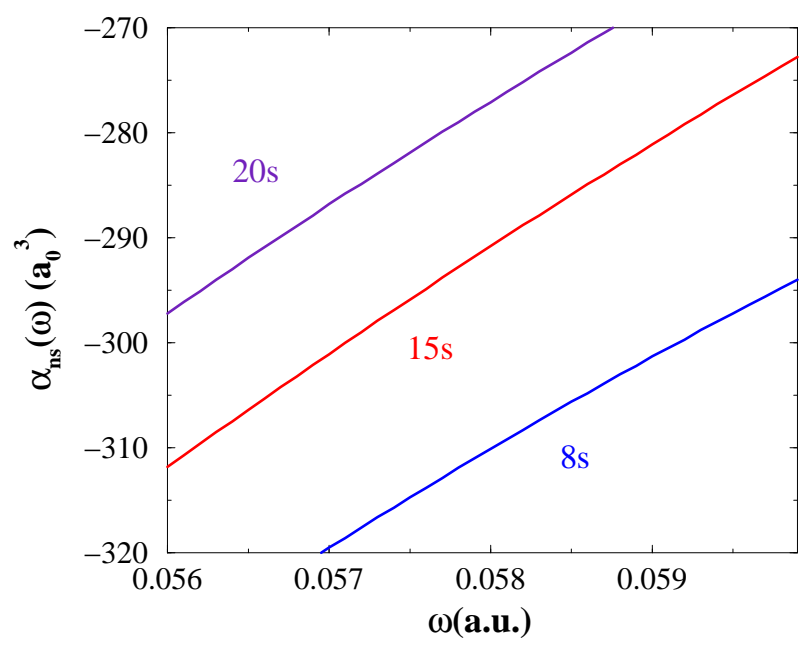

FIG. 1: Dynamic polarizability $\alpha(\omega)$ for the $n s$ states of Rb in atomic units.

Hartree-Fock value. We note that substituting HF energy by experimental values and leaving matrix elements unchanged yields substantially different $\left(-356 a_{0}^{3}\right)$ value, thus confirming our conclusion that using HF values for both matrix elements and energies produces more accurate values then replacing $\mathrm{HF}$ energies by experimental results. We note that current accuracy of $\alpha_{n s}(\omega)$ (estimated at $10 \%$ ) is sufficient for the purpose of present paper since the polarizability of the ground state varies vary rapidly with $\omega$ as illustrated in Table 1 and the variation of the value $\omega$ within the uncertainty of the Rydberg state polarizability is small. Consequently, the exact point where $\alpha_{5 s}(\omega)=\alpha_{n s}(\omega)$ can be determined experimentally by detuning of the lattice frequency near the matching point. Further investigations of the Rydberg state polarizabilities are beyond the scope of this paper 


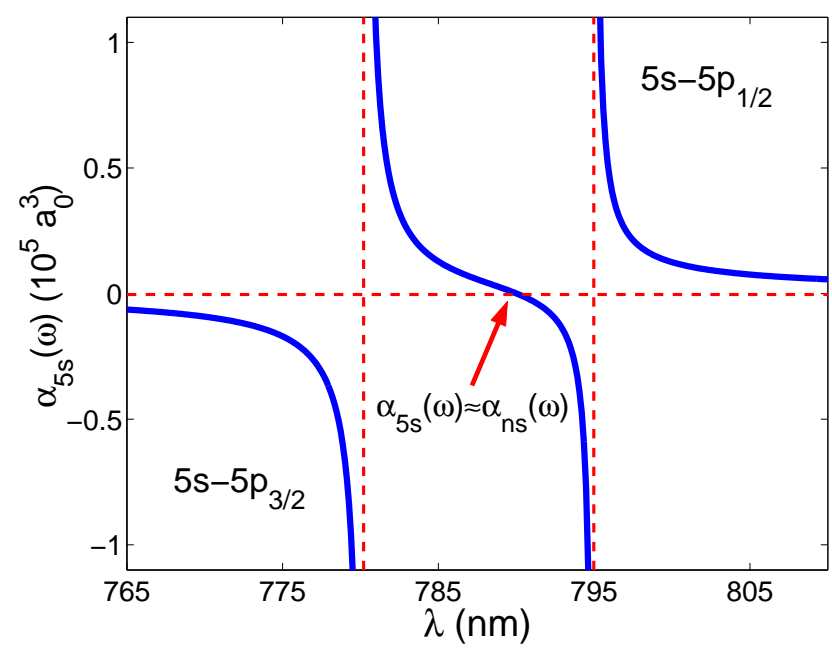

FIG. 2: Dynamic polarizability $\alpha(\omega)$ for the ground state of $\mathrm{Rb}$ in a.u. The horizontal dashed line corresponds to polarizability of the Rydberg ns level.

and will be considered elsewhere [22].

Finally, we investigate the dependence of the Rydberg state polarizabilities on the value of $\omega$ in the vicinity of the $5 s-5 p_{1 / 2}$ and $5 s-5 p_{3 / 2}$ resonances. As noted above, the polarizability of $n s$ state with $n>8$ does not change significantly with $n$. The results of the calculation of valence dynamic polarizability for the $8 s, 15 s$, and $20 s$ states with $\omega=0.056-0.060$ a.u. are illustrated in Fig. 1. We find that the dynamic polarizabilities of the Rydberg $n s$ states vary very weakly with $\omega$.

Summarizing the results above we find that the values of the ground state and Rydberg $n s$ state polarizabilities can be matched near the point between $5 s-5 p_{1 / 2}$ and $5 s-5 p_{3 / 2}$ resonances where the ground state polarizability changes sign as demonstrated in Fig. 2. The horizontal dashed line corresponds to polarizability of the Rydberg $n s$ level. The resonances are shown by the vertical dashed lines for clarity as we assumed $\omega$ to be a few linewidths from the resonances. We predict that value of $\omega$ where these polarizabilities match equals to $\lambda_{\text {vac }}=790.14(2) \mathrm{nm}$ for $15 \mathrm{~s}$ state. This value includes $10 \%$ uncertainty in the value of the 15 s state polarizability and $6 \%$ uncertainty in the value of the ground state polarizability. The next point at which the polarizabilities can be matched is close to the $5 s-6 p_{1 / 2}$ resonance which requires much larger detuning. The above discussion is valid for higher Rydberg states, which may be chosen for the gate implementation because of the longer lifetimes [24], since we found Rydberg state ac polarizability to vary weakly with the principal quantum number.

Next, we consider an alternative approach to the issue of the matching polarizabilities of the ground and arbitrary Rydberg state. This discussion is not limited to $n s$ Rydberg states. It follows from the expression for the dynamic polarizability that if the energy difference
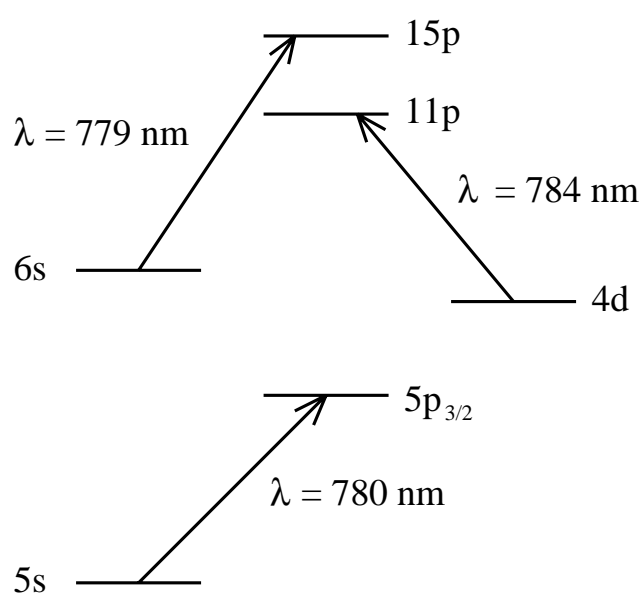

FIG. 3: Levels with transitions energies near $5 s-5 p_{3 / 2}$ resonance. Levels are not to scale.

$E_{n s}-E_{n^{\prime} p}$ or $E_{n d}-E_{n^{\prime} p}$ is accidentally close to the value of either $5 s-5 p_{1 / 2}$ or $5 s-5 p_{3 / 2}$ transition energy then the polarizability of the corresponding $s, p$, or $d$ state can be made large by detuning to the appropriate frequency. We have investigated the spectrum of Rb to locate energy differences which are close to the resonance $5 s-5 p_{1 / 2}$ or $5 s-5 p_{3 / 2}$ transition energies. The closest matches are $6 s-15 p$ and $4 d-11 p$ transition energies which differ from the $\delta E=E_{5 s}-E_{5 p_{3 / 2}}$ by $20 \mathrm{~cm}^{-1}$ and $60 \mathrm{~cm}^{-1}$, respectively. The corresponding energy level scheme is illustrated in Fig. 3. However, in both of these cases the terms with such denominators contribute to the polarizabilities of the $p$ states. The required detuning in this case is smaller but only $11 p$ and $15 p$ levels may be used in this method. We note that there are no such Na levels for the situation where the lattice light is near $\mathrm{Na}(3 \mathrm{p})$ levels. The advantage of this scheme is high value of the ac polarizabilities at the matching point; in the previous scheme the value of the ac polarizability at the matching point is relatively small, leading to the higher laser power requirement and subsequent higher scattering rate (on the order of $10 \mathrm{~s}^{-1}$ ). The disadvantage of using $n p$ vs. ns states in a Rydberg gate scheme is that excitation of transitions from the ground state requires either ultraviolet radiation or a three-photon process. Another difficulty of this approach is the necessity of the very fast gate operation times to achieve high gate fidelity because of the short, $4 \mu s$ [25], lifetime of the $15 p$ level.

We note that by choosing the appropriate Rydberg level and a longer wavelength trapping laser (e.d. $\mathrm{CO}_{2}$ laser) it should often be possible to find a matching scheme. In these situations, because of the number of nearby resonances for the Rydberg state, one probably would have to consider contributions from several intermediate states.

In summary, we have found two ways to match frequency-dependent polarizabilities of the Rydberg state 
and the ground state for optical lattices tuned near the $\mathrm{Rb}(5 \mathrm{p})$ states. First, the value of the ground state polarizability at $\lambda_{v a c}=790.032(8) \mathrm{nm}$ crosses zero and changes sign. Therefore, it can be matched with polarizabilities of various Rydberg levels near that point as demonstrated in Fig. 2. For the case of $15 \mathrm{~s}$ state, polarizabilities match at $\lambda_{v a c}=790.14(2) \mathrm{nm}$. Second, the value of $\omega$ can be detuned to nearly match the values of the $15 p-6 s$ and $11 p-4 d$ transition energies which are accidentally close to the $\delta E=E\left(5 p_{3 / 2}\right)-E(5 s)$ energy. Then, the dynamic polarizability of the $15 p$ or $11 p$ state can be made large enough to match the polarizability of the ground state near $5 p_{3 / 2}-5 s$ resonance. The matching of the frequency-dependent polarizabilities of the atom in its ground and Rydberg states results in the matching of the optical potential seen by the atom during the gate operation and provides the optimal scheme for the Rydberg gate operation with respect to the motional decoherence.

Another way of eliminating the differential in the trapping potential between the ground and Rydberg states is to switch the trap off during the time of the gate action, and turn it back on after the action is completed. This procedure also induces heating of the atomic center of mass motion, the effect of which can be characterized as follows. Suppose that, at time $t=0$, an atom of mass $M$ is in the ground state of a trap site, where for convenience we assume the potential to be that of an isotropic oscillator of frequency $\omega_{0}$, with ground state energy $E_{0}=3 \hbar \omega_{0} / 2$. If the trap is turned off suddenly, the wavefunction $\Psi\{\mathbf{r}, t\}$ for the center of mass coordinate $\mathbf{r}$ evolves during time $\mathrm{t}$ as

$$
\Psi\{\mathbf{r}, t\}=\frac{\exp \left(-r^{2} / 2 d_{0}^{2}\left(1+i \omega_{0} t\right)\right)}{\pi^{3 / 4} d_{0}^{3 / 2}\left(1+i \omega_{0} t\right)^{3 / 2}}
$$

where $d_{0}=\sqrt{\hbar / M \omega_{0}}$. In free expansion of the atomic wavepacket from the released trap, its mean kinetic energy, $\langle T\rangle=E_{0} / 2$ remains constant, while its meansquare radius grows as $\left\langle r^{2}(t)\right\rangle=3 d_{0}^{2}\left(1+\omega_{0}^{2} t^{2}\right) / 2$. If the trap is suddenly turned back on again at time $\tau$, the mean energy of the wavepacket in the restored trapping potential is $E=E_{0} / 2+M \omega_{0}^{2}<r^{2}(\tau)>/ 2=E_{0}\left(1+\omega_{0}^{2} \tau^{2} / 2\right)$. This corresponds to a heating of $k_{\mathrm{B}} T=\left(\hbar \omega_{0}\right) \omega_{0}^{2} \tau^{2} / 4$ per cycle of trap release and restoration. Such heating can be avoided by use of a matched potential scheme of the type proposed here. For a trap frequency of a $1 \mathrm{MHz}$ and a $1 \mu$ s gate time one obtains $k_{B} T \approx 0.006\left(\hbar \omega_{0}\right)$. The gate operation time in principle can be optimized by appropriate choice of the Rydberg level, the applied dc field, and laser power.

We gratefully acknowledge discussions with Steven Rolston, Wendell Hill, Walter Johnson, Philippe Grangier, Mark Saffman, and Thad Walker. This work was partially supported by the Advanced Research Development Activity, the National Security Agency, and NIST Advanced Technology Program.
[1] D. Jaksch, H.-J. Briegel, J. I. Cirac, C. W. Gardiner, and P. Zoller, Phys. Rev. Lett. 82, 1975 (1999).

[2] T. Calarco, E. A. Hinds, D. Jaksch, J. Schmiedmayer, J. I. Cirac, and P. Zoller, Phys. Rev. A 61, 022304 (2000).

[3] H.-J. Briegel, T. Calarco, D. Jaksch, J. I. Cirac, P. Zoller, et al. J. Mod. Opt. 47, 415 (2000).

[4] E. Charron, E. Tiesinga, F. Mies, C.J. Williams, Phys. Rev. Lett. 88, 077901 (2002).

[5] G. K. Brennen, C. M. Caves, P. S. Jessen, and I. H. Deutsch, Phys. Rev. Lett. 82, 1060 (1999).

[6] G K. Brennen and I. H. Deutsch, Phys. Rev. A 61, 062309 (2000).

[7] G.E. Brennan, I.H. Deutsch, C.J. Williams, Phys. Rev. A 65, 022313 (2002).

[8] D. Jaksch, J.I. Cirac, P. Zoller, S.L. Rolston, R. Côté, and M.D. Lukin, Phys. Rev. Lett. 85, 2208 (2000).

[9] I.E. Protsenko, G. Reymond, N. Schlosser, and P. Grangier, Phys. Rev. A 65, 052301 (2002).

[10] P. Avan, C. Cohen-Tannoudji, J. Dupont Roc, and C. Fabre, J. Phys. (Paris) 37, 993 (1976)

[11] A. Derevianko, W. R. Johnson, M. S. Safronova, and J. F. Babb, Phys. Rev. Lett. 82, 3589 (1999).

[12] W. R. Johnson, D. Kolb, and K.-N. Huang, Atomic Data Nuc. Data Tables 28, 333 (1983).

[13] I. Johansson, Ark. f. Fysik 20, 135 (1961).

[14] M. S. Safronova, W. R. Johnson, and A. Derevianko,
Phys. Rev. A 60, 4476 (1999).

[15] U. Volz and H. Schmoranzer, Phys. Scr. T65, 48 (1996)

[16] M.S. Safronova, Ph.D. Thesis, University of Notre Dame (2001).

[17] C.E. Moore, Atomic Energy Levels, Natl. Bur. Stand. Ref. Data Ser., Natl. Bur. Stand. (U.S.) Circ. No. 35 (U.S. GPO, Washington, D.C., 1971), Vols. II.

[18] R. Molof, H.L. Schwartz, T.M. Miller, and B. Bederson, Phys. Rev. A 10, 1131 (1974).

[19] M. Marinescu, H.R. Sagedghpour, and A. Dalgarno, Phys. Rev. A 49, 5103 (1994).

[20] We use the value $\omega=0.04298$ a.u. that was used in Ref. [19], but we note that the value $\omega=0.04281$ a.u. actually corresponds to the wavelength in air of $\lambda=1.064 \mu$ of the $\mathrm{Nd}$ :YAG laser.

[21] K.D. Bonin and M.A. Kadar-Kallen, Phys. Rev. A47, 944 (1993).

[22] M.S. Safronova, C. J. Williams, and C. W. Clark, unpublished.

[23] W. R. Johnson, S. A. Blundell, and J. Sapirstein, Phys. Rev. A37, 2764 (1988).

[24] A.L. de Oliveira, M.W. Mancini, V.S. Bagnato, and L.G. Marcassa, Phys. Rev. A 65, 031401 (2002)

[25] C.E. Theodosiou, Phys. Rev. A 30, 2881 (1984) 